The Astronomical Journal, 129:2849-2855, 2005 June

(C) 2005. The American Astronomical Society. All rights reserved. Printed in U.S.A.

\title{
MULTIPLICITY AMONG WIDELY SEPARATED BROWN DWARF COMPANIONS TO NEARBY STARS: GLIESE 337CD
}

\author{
Adam J. Burgasser, ${ }^{1,2}$ J. Davy Kirkpatrick, ${ }^{3}$ and Patrick J. Lowrance ${ }^{4}$ \\ Received 2004 December 20; accepted 2005 March 13
}

\begin{abstract}
We present Lick Natural Guide Star Adaptive Optics observations of the L8 brown dwarf G1 337C, which is resolved for the first time into two closely separated ( $0.53 \pm 0.03)$, nearly equal magnitude components with a $K_{s}$ flux ratio of $0.93 \pm 0.10$. Companionship is inferred from the absence of a 3".6 offset source in Two Micron All Sky Survey or photographic plate images, implying that the observed secondary component is a comoving late-type dwarf. With a projected separation of 11 AU and nearly equal magnitude components, Gl 337CD has properties similar to those of other known companion and field substellar binaries. Its long orbital period (estimated to be $\sim 140-$ $180 \mathrm{yr}$ ) inhibits short-term astrometric mass measurements, but the Gl 337CD system is ideal for studying the L-T transition at a fixed age and metallicity. From a compilation of all known widely separated ( $\gtrsim 100 \mathrm{AU})$ stellarbrown dwarf multiple systems, we find evidence that the binary fraction of brown dwarfs in these systems is notably higher than that of field brown dwarfs, $45_{-13}^{+15} \%$ versus $18_{-4}^{+7} \%$ for analogous samples. We speculate on possible reasons for this difference, including the possibility that dynamic (ejection) interactions that may form such wide pairs preferentially retain binary secondaries because of their greater combined mass and/or ability to absorb angular momentum.
\end{abstract}

Key words: binaries: visual — stars: individual (G1 337CD) — stars: low-mass, brown dwarfs

\section{INTRODUCTION}

Multiple systems are important laboratories for studying the physical properties and origins of brown dwarfs, low-mass stars incapable of sustaining core hydrogen fusion (Kumar 1962; Hayashi \& Nakano 1963). Brown dwarf binaries facilitate the study of mass or temperature effects in cool atmospheres independent of age or metallicity variations and can be astrometrically and/or spectroscopically monitored to yield system mass measurements (e.g., Zapatero Osorio et al. 2004). Substellar companions to well-characterized main-sequence stars inherit the age and metallicity of the primary, assuming coevality, providing additional constraints on mass and composition (e.g., Burgasser et al. 2000). Finally, the multiplicity fraction, separation distribution, and mass ratio distribution of brown dwarf binaries and brown dwarf companions provide important clues for the mechanism of their formation (e.g., Close et al. 2003).

Brown dwarfs in multiple systems have been found largely in two distinct populations: as widely separated ( $\rho \gtrsim 20 \mathrm{AU})$ companions to stars, forming low mass ratio systems $\left(q \equiv M_{2} / M_{1} \sim\right.$ 0.1 ), and as closely separated ( $\rho \lesssim 20 \mathrm{AU}$ ) binaries in systems with near-unity mass ratios. The paucity of closely separated brown dwarf companions, most evident in radial velocity monitoring programs, has been termed the "Brown Dwarf Desert" (Marcy \& Butler 2000), and a number of mechanisms have been postulated to explain this trend (e.g., Armitage \& Bonnell 2002). On the other hand, the apparent absence of comoving

\footnotetext{
${ }^{1}$ Department of Astrophysics, Division of Physical Sciences, American Museum of Natural History, Central Park West at 79th Street, New York, NY 10024; adam@amnh.org.

2 Spitzer Fellow.

3 Infrared Processing and Analysis Center, California Institute of Technology, Mail Stop 100-22, Pasadena, CA 91125; davy@ipac.caltech.edu.

4 Spitzer Science Center, California Institute of Technology, Mail Stop 220-6, Pasadena, CA 91125; lowrance@ipac.caltech.edu.
}

widely separated brown dwarf pairs ${ }^{5}$ has been seen as support for dynamical ejection models for brown dwarf formation (e.g., Bate et al. 2002a).

A third group of substellar multiple systems shares the properties of both of these classes: closely separated brown dwarf binaries that are widely separated but gravitationally bound to more massive stellar primaries. Six such systems are currently known: Gl 569Bab (Forrest et al. 1988; Martín et al. 2000), GJ 1001BC (Goldman et al. 1999; Golimowski et al. 2004b), Gl 577BC (Lowrance 2001; McCarthy et al. 2001; Mugrauer et al. 2004; Lowrance et al. 2005), Gl 417BC (Kirkpatrick et al. 2001a; Bouy et al. 2003), HD 130948BC (Potter et al. 2002; Goto et al. 2002), and $\epsilon$ Ind BC (Scholz et al. 2003; McCaughrean et al. 2004). All six binaries have high mass ratios $(q \gtrsim 0.6)$ and are separated by at least $45 \mathrm{AU}$ from their apparently single and more massive main-sequence primaries. One of these systems, Gl 569Bab, has been astrometrically and spectroscopically monitored over a few orbital periods, providing the first mass measurements for a substellar system (Lane et al. 2001; Zapatero Osorio et al. 2004); follow-up observations of HD 130948BC and $\epsilon$ Ind BC are currently under way (D. Potter \& M. McCaughrean 2004, private communication). By providing multiple, independent constraints on substellar masses and ages, these companion binaries provide powerful empirical tests of brown dwarf evolutionary and structural models (Lane et al. 2001).

In this article we present the discovery of a new binary brown dwarf companion system, Gl 337CD, identified through highresolution imaging observations obtained with the Lick Observatory adaptive optics (AO) system. In $\S 2$ we describe our observations and data reduction techniques. In $\S 3$ we analyze

\footnotetext{
${ }^{5}$ Luhman (2004) has reported the identification of a widely separated ( $\rho=240 \mathrm{AU}$ ) brown dwarf pair in the Cha I star-forming region, based on their low-gravity spectra (implying common cluster membership) and statistically significant angular proximity. However, common motion for these objects has not been verified, and their physical association remains uncertain.
} 
the results, deriving physical properties for the binary and probable orbital characteristics. We also argue for physical association of the brown dwarf pair based on the proper motion of the Gl 337 system. In $\S 4$ we discuss the wider issue of multiplicity among brown dwarf companions, identifying a significantly higher binary fraction among these sources as compared to field brown dwarfs. We speculate on how this may be related to the formation of wide binary systems. Results are summarized in $\S 5$.

\section{OBSERVATIONS}

The combined system G1 337C was originally identified as the unresolved source 2MASSW J0912145+145940 by Wilson et al. (2001) in the Two Micron All Sky Survey (2MASS; Cutri et al. 2003). Optical spectroscopy indicates a spectral type of L8, sufficiently late to deduce that this source is substellar. G1 337C is a widely separated ( $\left.\rho=43^{\prime \prime} \sim 880 \mathrm{AU}\right)$ common proper motion companion to the G1337AB system, a G8 V+K1 V (Mason et al. 1996; Richichi et al. 2000; Barnaby et al. 2000), nearly equal mass (Pourbaix 2000), double-lined spectroscopic and visual binary with an orbital separation of $2.4 \mathrm{AU}$ and a period of $2.7 \mathrm{yr}$ (Mason et al. 1996). The system lies at a distance of $20.5 \pm 0.4 \mathrm{pc}$ from the Sun (Perryman et al. 1997). Wilson et al. (2001) estimate an age of 0.6-3.4 Gyr for Gl 337AB on the basis of X-ray luminosity and kinematics. The absolute $J H K_{S}$ magnitudes of G1 337C are 0.4-0.6 mag brighter than those of several L8 dwarfs with parallax measurements (Dahn et al. 2002; Vrba et al. 2004), and this source is 0.8 mag brighter at the $J$ band than the L8 companion brown dwarf G1 584C (Kirkpatrick et al. 2000). These measurements have suggested that Gl 337C could be an unresolved multiple system.

We observed G1 337C as part of a backup natural guide star program during a single-night run with the Lick Observatory AO system on 2004 February 9 (UT). A log of observations is given in Table 1. The Lick-Lawrence Livermore National Laboratory (LLNL) AO system on the $3 \mathrm{~m}$ Shane Telescope uses the IRCAL near-infrared imager (Lloyd et al. 2000) behind a 127 -actuator (61 actively controlled) deformable mirror. Corrections to the mirror surface are made through observation of a $V \lesssim 12-13$ guide star within $55^{\prime \prime}$ of the target source onto a Shack-Hartmann wavefront sensor and fast-read CCD camera. A photodiode quad cell provides tip-tilt corrections. At reasonable Strehl ratios, diffraction-limited $\left(\sim 00^{\prime \prime} 12\right.$ at $\left.K_{s}\right)$ cores of point sources can be marginally resolved with the $00^{\prime \prime} 076$ pixel resolution of IRCAL. The imaging field of view is 19".4 on a side. Additional information on the Lick AO system is given in Bauman et al. (1999, 2002) and Gavel et al. (2000).

Conditions during the night were poor, with seeing of $\sim 1^{\prime \prime}$ at the $K$ band and intermittent clouds and cirrus. We used the bright Gl 337AB pair (combined $V=6.78$ ) as our tip-tilt and AO corrector source; its binarity did not affect the AO correction due to the $2^{\prime \prime}$ pixel scale of the wavefront sensor. Five $120 \mathrm{~s}$ dithered exposures of G1 $337 \mathrm{C}$ were obtained at $K_{s}$, and three $300 \mathrm{~s}$ dithered exposures were obtained in the narrowband $K_{2.2}$ filter centered at $2.2 \mu \mathrm{m}(\Delta \lambda=0.02 \mu \mathrm{m})$. Because of the poor and variable seeing $\left(r_{0} \approx 5-15 \mathrm{~cm}\right)$ and wide separation between the AO corrector and science target, Strehl ratios were low over the course of the observations $(\sim 0.06)$. The point-spread function (PSF) of the observations was measured by imaging the single source USNO-B1.0 0992-0177063 (Monet et al. 2003) in both filters while guiding on the $R=8.1$ guide star USNO-B1.0 0992-0177081. This pair has a similar separation and position angle $\left(\rho=45^{\prime \prime} .3\right.$ at $\left.\theta=276^{\circ}\right)$ to that between Gl 337AB and Gl 337C $\left(\rho=46^{\prime \prime} 0\right.$ at $\left.\theta=262^{\circ}\right)$. The PSF FWHM was $0 . \prime 45$.
Imaging data were reduced by first constructing dark-subtracted, median-combined, and normalized flat-field images for both filters from observations of the twilight sky at the start of the evening. The sky flat and dark frames were also used to identify dead and hot pixels for the construction of a pixel mask. Observations of the science targets were pairwise subtracted (using sequential imaging pairs) to eliminate sky background, then divided by the flat-field frame and corrected for bad pixels by linear interpolation. Dithered pairs were combined by shifting (in integer pixel units) and adding, centering on the peak of each source as verified by visual inspection.

Reduced images for Gl $337 \mathrm{C}$ and its associated PSF star in both $K_{s}$ and $K_{2.2}$ filters are shown in Figure 1. The extended nature of Gl 337C is clearly evident along an east-southeastwest-northwest axis. The PSFs of the components are heavily blended, however, as a result of the poor AO correction during the observations. These images were deconvolved using the PSF-fitting code XPHOT, kindly provided by D. Koerner. Starting from the observed PSF of the single star and estimates of the centers of the components, this code compares model images to the binary data by iteratively shifting the relative positions and peak fluxes of the two components. Convergence occurs when the standard deviation of the difference image between the data and model image is minimized. Multiple fits to the $x$ and $y$ pixel separations and flux ratio between the components were obtained by using each of the individual PSF observations (reduced dithered pairs) and varying the initial starting conditions. The scatter in these fits was used as an estimate of the measurement uncertainty of the mean value.

For astrometric calibration, we observed the visual double HD 56988AB, selected from Shatsky et al. (1999). This pair has a known separation $\left(\rho=3^{\prime \prime} .992 \pm 0.004\right)$ and position angle $\left(\theta=350^{\circ} .83 \pm 0.10\right)$ ideally suited for observation with IRCAL. Both components of this double were bright enough to serve as AO corrector stars ( $V \approx 9.0$ for each component), and we used the southernmost source for this purpose. Five dithered exposures each were obtained at $K_{s}$ and $K_{2.2}$. Images were reduced as described above. We used XPHOT to measure the separation of the pair, again employing multiple PSFs (in this case, each component of the double) and initial starting conditions to estimate the empirical uncertainty. From these measurements, we derived the image pixel scale $\left(00^{\prime \prime} 076 \pm 0\right.$."005) and orientation $(1.35 \pm 0.15$ east of north) of the camera.

\section{ANALYSIS}

\subsection{Common Proper Motion}

While the PSF analysis clearly indicates the presence of two sources at the position of G1 337C, are these sources physically associated? The G1 337 system has a proper motion $\mu=$ 0 .'5789 \pm 0 ".0009 $\mathrm{yr}^{-1}$ (Perryman et al. 1997), implying that G1 337C itself has moved 3". 6 between the time of its detection by 2 MASS (1997 November 18 UT) and our observations. If the second component identified in our observations were an unassociated stationary background source, it would have been readily detected as a separate point source by $2 \mathrm{MASS}$. We performed overlapping PSF simulations on the 2MASS atlas images of G1 337C to determine that an equal-magnitude pair can be resolved in those data for separations of $\sim 1^{\prime \prime} .5$ and greater. ${ }^{6}$ This limit allows us to constrain the motion of the second component to $0.33-0.81 \mathrm{yr}^{-1}$ and a position angle within $\pm 25^{\circ}$ of

\footnotetext{
6 2MASS point-source extraction generally resolves sources only down to $5^{\prime \prime}$ as a result of blending (Cutri et al. 2003, § I.6.b.ix).
} 
TABLE 1

Log of AO Observations on 2004 February 9 (UT)

\begin{tabular}{|c|c|c|c|c|c|c|c|c|c|c|c|}
\hline Target & $\begin{array}{c}\alpha \\
(\mathrm{J} 2000.0)\end{array}$ & $\begin{array}{c}\delta \\
(\mathrm{J} 2000.0)\end{array}$ & $K_{s}^{\mathrm{a}}$ & $\begin{array}{c}\mathrm{AO} \\
\text { Corrector }\end{array}$ & $\Delta \alpha^{\mathrm{b}}$ & $\Delta \delta^{\mathrm{b}}$ & $R^{\mathrm{c}}$ & UT & Filter & $\begin{array}{c}t \\
(\mathrm{~s})\end{array}$ & Air Mass \\
\hline HD 56988AB & 071949.31 & +132223.0 & $8.81 \pm 0.02^{\mathrm{d}}$ & HD 56988B & & . & $9.2^{\mathrm{d}}$ & $\begin{array}{l}04: 19 \\
04: 27\end{array}$ & $\begin{array}{c}K_{s} \\
K_{2.2}\end{array}$ & $\begin{array}{r}25 \\
150\end{array}$ & $\begin{array}{l}1.21 \\
1.18\end{array}$ \\
\hline G1 337CD & 091214.69 & +14 5939.6 & $14.04 \pm 0.06$ & Gl 337AB & -42.6 & -5.8 & 6.0 & $\begin{array}{l}06: 05 \\
06: 20\end{array}$ & $\begin{array}{c}K_{s} \\
K_{2.2}\end{array}$ & $\begin{array}{l}600 \\
900\end{array}$ & $\begin{array}{l}1.21 \\
1.18\end{array}$ \\
\hline USNO-B1.0 0992-0177063 .................. & 083826.49 & +091658.5 & $10.18 \pm 0.02$ & USNO-B1.0 0992-0177081 & $-44^{\prime \prime} 4$ & $4 " 8$ & 8.1 & $\begin{array}{l}06: 41 \\
06: 47\end{array}$ & $\begin{array}{c}K_{2.2} \\
K_{s}\end{array}$ & $\begin{array}{l}240 \\
100\end{array}$ & $\begin{array}{l}1.16 \\
1.15\end{array}$ \\
\hline
\end{tabular}

NoтE.- - Units of right ascension are hours, minutes, and seconds, and units of declination are degrees, arcminutes, and arcseconds.

From 2MASS (Cutri et al. 2003).

Offset from AO corrector star to science target in arcseconds.

c From USNO-B1.0 (Monet et al. 2003).

d $R$ magnitude for the A component. 

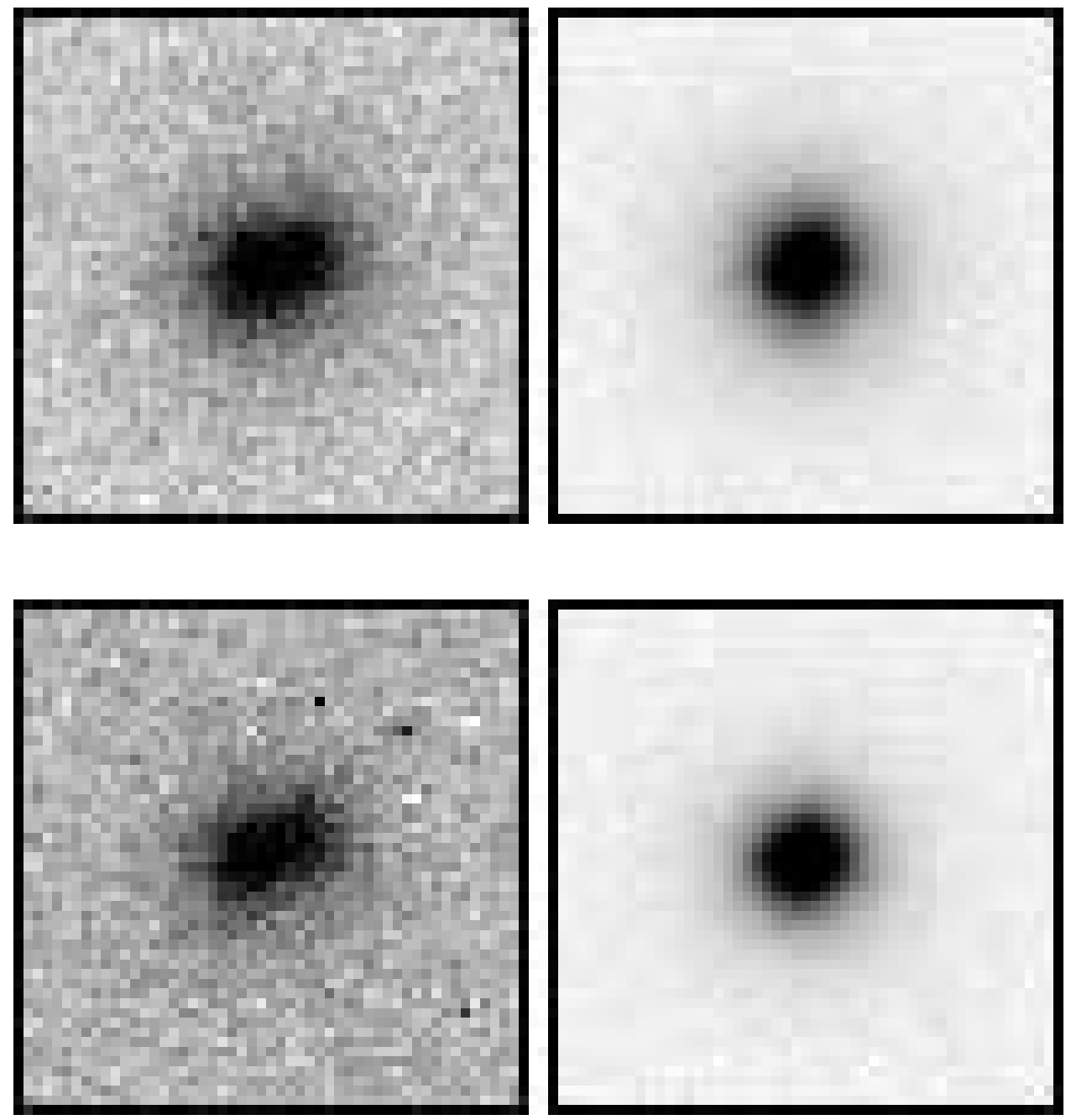

FIG. 1.-IRCAL images of G1 337CD (left) and the PSF star USNO-B1.0 0992-0177063 (right) in the $K_{s}($ top $)$ and $K_{2.2}$ (bottom) filters. Images are 3.' 9 on a side and are oriented with north to the top and east to the left. The two components of Gl $337 \mathrm{CD}$ are marginally resolved at $0.53 \pm 0.03$ separation, with the west-northwest component being slightly fainter at $K_{2.2}$.

that of Gl 337C. These constraints rule out an extragalactic source for the second component and strongly suggest common proper motion.

In addition, there are no optical sources coincident with or moving toward the current G1 337C position in the First or Second Palomar Observatory Sky Survey (POSS) plates (Wilson 1952; Reid et al. 1991). The second component must therefore have very red optical/near-infrared colors $\left(R-K_{s} \gtrsim 5\right)$, consistent with a late-type star or brown dwarf. These constraints on the motion and color of the resolved double, along with the angular proximity of the components, lead us to conclude that they comprise a gravitationally bound pair. We hereafter refer to the combined system as Gl 337CD.

\subsection{Properties of $\mathrm{Gl} 337 C D$}

Table 2 lists the derived separation, position angle, and $K_{s}$ and $K_{2.2}$ flux ratios for G1 $337 \mathrm{CD}$. Derived flux ratios were $0.93 \pm 0.10$ at $K_{s}$ and $0.90 \pm 0.08$ at $K_{2.2}$, with the westnorthwest component being slightly fainter in the latter filter. However, both measurements are consistent with equal brightness at the $K$ band, implying that G1 337CD is likely to be a near equal mass binary, $q \sim 1$, similar to the $\mathrm{AB}$ components. Assuming equal brightness across the near-infrared, the individual absolute 2MASS magnitudes of G1 $337 \mathrm{C}$ and D are

TABLE 2

Properties of G1 337CD

\begin{tabular}{|c|c|c|}
\hline Parameter & Value & Reference \\
\hline $\mathrm{SpT}^{\mathrm{a}}$ & L8 & 1 \\
\hline$d \ldots \ldots \ldots \ldots$ & $20.5 \pm 0.4 \mathrm{pc}$ & 2 \\
\hline$\mu$ & $0.5789 \pm 0^{\prime \prime} 0009 \mathrm{yr}^{-1}$ & 2 \\
\hline Age .............. & $0.6-3.4 \mathrm{Gyr}$ & 1 \\
\hline \multirow[t]{2}{*}{$\rho_{\mathrm{AB}-\mathrm{CD}} \cdots \ldots \ldots \ldots \ldots \ldots \ldots \ldots$} & $43^{\prime \prime}$ & 1 \\
\hline & $880 \mathrm{AU}$ & 1 \\
\hline \multirow[t]{2}{*}{$\rho_{\mathrm{C}-\mathrm{D}} \ldots \ldots \ldots \ldots \ldots \ldots \ldots \ldots \ldots$} & $0.53 \pm 0.03$ & 3 \\
\hline & $10.9 \pm 0.6 \mathrm{AU}$ & 3 \\
\hline$\theta_{\mathrm{C}-\mathrm{D}} \ldots \ldots \ldots \ldots \ldots \ldots \ldots \ldots \ldots \ldots$ & $291^{\circ} \pm 8^{\circ}$ & 3 \\
\hline$\Delta K_{s}$ & $0.08 \pm 0.12 \mathrm{mag}$ & 3 \\
\hline$\Delta K_{2.2} \ldots \ldots \ldots \ldots \ldots \ldots \ldots$ & $0.11 \pm 0.10 \mathrm{mag}$ & 3 \\
\hline 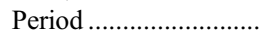 & $\sim 140-180 \mathrm{yr}$ & 3 \\
\hline
\end{tabular}

${ }^{a}$ Optical spectral type from Wilson et al. (2001).

ReFERENCES.- (1) Wilson et al. 2001; (2) Perryman et al. 1997; (3) this paper. 
$M_{J}=14.89 \pm 0.09, M_{H}=13.78 \pm 0.09$, and $M_{K_{s}}=13.21 \pm$ 0.07 . These values are consistent with those of G1 584C and the field object 2MASS $1632+1904$, both classified in the optical as L8 dwarfs (Kirkpatrick et al. 1999b; Dahn et al. 2002; Vrba et al. 2004). However, Vrba et al. (2004) have shown that $M_{K_{\mathrm{s}}}$ is effectively constant $(\sim 13.5 \pm 0.5)$ from L8 to T4, implying that the secondary could in fact be an early- or mid-type T dwarf. Resolved imaging and/or spectroscopy can test this possibility.

The astrometric calibrations yield consistent separations of $0.53 \pm 0.03$ at $292^{\circ} \pm 7^{\circ}$ in the $K_{s}$ images and $0.52 \pm 0.03$ at $290^{\circ} \pm 8^{\circ}$ at $K_{2.2}$. The uncertainty is dominated by scatter among the PSF fits. These values give a projected separation $\rho=10.9 \pm 0.6 \mathrm{AU}$, on the high end of the separation distribution among known brown dwarf binaries (Bouy et al. 2003; Burgasser et al. 2003; Gizis et al. 2003) and implying a long orbital period. Using the same mass estimates from Wilson et al. (2001) of $0.04 M_{\odot} \lesssim M \lesssim 0.07 M_{\odot}$ for each component, and assuming a semimajor axis $a \approx 1.26 \rho$ (Fischer \& Marcy 1992), we estimate an orbital period of 140-180 yr. Hence, this system is not an ideal target for astrometric monitoring, although it is amenable to resolved photometric and spectroscopic investigations.

Assuming reasonable values for the orbital eccentricity of the CD pair $(\epsilon \lesssim 0.5)$, the G1 337ABCD system is dynamically stable as long as the AB-CD orbital eccentricity is less than 0.8-0.9 (Eggleton \& Kiseleva 1995). While the wide separation between the binaries suggests that a highly elliptical orbit is possible, it is more likely that this system is a long-lived dynamical arrangement.

\section{DISCUSSION}

\subsection{The Binary Fraction of Widely Separated Brown Dwarf Companions}

G1 337CD joins a growing list of binary brown dwarf systems that are widely separated, common motion companions to stellar primaries. To place this system in context, we compare its properties to those of other known, widely separated, common motion brown dwarf companions, single or binary (Table 3 ). We adopt a lower separation limit of $100 \mathrm{AU}$ for this list, similar to limits used for most searches of widely separated systems (e.g., Hinz et al. 2002). This constraint excludes the companion doubles G1 569Bab and HD 130948BC, a handful of unresolved brown dwarf companions, and planets/brown dwarfs identified through radial velocity techniques. Nevertheless, the 17 brown dwarf systems listed in Table 3 comprise a useful sample for exploring the properties of widely separated brown dwarf companions to nearby stars.

Several characteristics of the substellar companion binaries are similar to those of substellar field binaries. Both groups exhibit short projected separations $(\rho<20 \mathrm{AU})$ and near-unity component mass ratios. The distribution of separations is also similar, peaking around 2-4 AU (Bouy et al. 2003; Gizis et al. 2003) for both groups. However, the fraction of binaries $\left(\epsilon_{b} \equiv N_{\text {binary }} / N_{\text {total }}\right)$ is higher among the companions. For all sources listed in Table $3, \epsilon_{b}=29_{-8}^{+13 \%}$ (five binaries out of 17 total), ${ }^{7}$ somewhat larger than the binary fraction typical for field samples, $\epsilon_{b} \approx 10 \%-20 \%$ (Reid et al. 2001; Bouy et al. 2003; Burgasser et al. 2003; Close et al. 2003; Gizis et al. 2003). Restricting both samples to only brown dwarfs (spectral types L4 and later among the field objects; Gizis et al. 2000) with

\footnotetext{
${ }^{7}$ Statistical uncertainties for $\epsilon_{b}$ are computed following the prescription of Burgasser et al. (2003)
}

Hubble Space Telescope or AO observations, the binary fraction gap widens: $\epsilon_{b}=45_{-13}^{+15 \%}$ (five out of 11) for the companion brown dwarfs versus $18_{-4}^{+7} \%$ (nine out of 50) for the field brown dwarfs, a $2 \sigma$ deviation. An important caveat to this result is the fact that neither sample is a complete, unbiased, or volume-limited one. However, as the sources in both samples were generally identified by similar techniques (compiled from magnitude-limited, color-selected surveys, followed by highresolution imaging) and have similar distances and separation distributions, any selection biases are likely to be common. The dissimilarity in the binary fractions between companion and field brown dwarfs, at least in the parameter space explored by highresolution imaging, is therefore compelling.

What could account for the higher binary fraction among the widely separated companions? One current, albeit controversial, model for the formation of brown dwarfs proposes that early mass accumulation by protostars can be aborted by dynamic encounters with other prestellar cores and disks, causing ejection out of the nascent gas cloud (Reipurth \& Clarke 2001; Bate et al. 2002a). In such encounters, the greater the difference in mass between the scattering sources, the more likely it is that the less massive object (in this case, a brown dwarf or brown dwarf progenitor) will be scattered away from the more massive object. On the other hand, if the deflected object is a tight brown dwarf binary system, the larger combined mass of this pair could allow it to be gravitationally captured. This capture may be facilitated by the transfer of angular momentum into the brown dwarf binary orbit during the encounter. Presuming that momentum transfer does not disrupt the binary pair, such encounters may produce weakly bound, widely separated multiple systems more readily than those involving single brown dwarfs, leading to a binary excess among the widely separated companions. Observable consequences of this process may include wider and/ or more elliptical orbits for widely separated companion brown dwarf binaries, which can be tested as further examples of these systems are identified.

The possibility of a higher binary fraction for brown dwarf companions resonates with the observed enhanced frequency of spectroscopic binaries among components of visual multiples (Tokovinin \& Smekhov 2002). The presence of a third star is believed to be integral to the formation of such close pairs, as it removes angular momentum from the binary upon scattering (Tokovinin 1997; Bate et al. 2002b). This interaction is somewhat orthogonal to the mechanism described above, in which the lowmass binary gains angular momentum to remain bound in the wider system. However, the similarity of these processes suggests that both could potentially occur in the high stellar density environment of a star-forming region. Simulations exploring these interactions in detail are necessary to determine their feasibility. Regardless, it is interesting to speculate whether the G1 337ABCD system arose through the exchange of angular momentum between both of its double components.

\subsection{Gl 337CD and the L-T Transition}

While G1 337CD is too wide to adequately measure its full orbital characteristics in a reasonable period, this binary does provide a unique opportunity to study the properties of cool brown dwarf atmospheres, particularly across the transition between L dwarfs and T dwarfs. The dramatic shift in near-infrared spectral energy distributions between late-type L dwarfs, characterized by continuum dust emission and $\mathrm{H}_{2} \mathrm{O}$ and $\mathrm{CO}$ bands, and mid-type $\mathrm{T}$ dwarfs, characterized by an absence of dust and strong $\mathrm{H}_{2} \mathrm{O}$ and $\mathrm{CH}_{4}$ bands, appears to occur over a fairly narrow effective temperature range (Kirkpatrick et al. 2000; 
TABLE 3

Widely Separated Brown Dwarf Companion Systems

\begin{tabular}{|c|c|c|c|c|c|c|c|c|c|}
\hline \multirow[b]{2}{*}{ NAME } & \multicolumn{2}{|c|}{ Spectral Types } & \multirow{2}{*}{$\begin{array}{c}\rho_{*-\mathrm{BD}} \\
(\operatorname{arcsec})\end{array}$} & \multirow{2}{*}{$\begin{array}{l}\rho_{*-\mathrm{BD}} \\
(\mathrm{AU})\end{array}$} & \multirow{2}{*}{$\begin{array}{l}\rho_{\mathrm{BD}-\mathrm{BD}}{ }^{\mathrm{a}} \\
(\operatorname{arcsec})\end{array}$} & \multirow{2}{*}{$\begin{array}{c}\rho_{\mathrm{BD}-\mathrm{BD}}{ }^{\mathrm{a}} \\
(\mathrm{AU})\end{array}$} & \multirow[b]{2}{*}{$q$} & \multirow{2}{*}{$\begin{array}{c}\text { AGE } \\
\text { (Gyr) }\end{array}$} & \multirow[b]{2}{*}{ REFERENCE } \\
\hline & Primaries & Secondaries & & & & & & & \\
\hline TWA 5B & $\mathrm{M} 1.5 \mathrm{~V}$ & $\mathrm{M} 8.5 \mathrm{~V}$ & 2.0 & 100 & $\lesssim 0.15$ & $\lesssim 8$ & $\ldots$ & $0.02-0.3$ & 1,2 \\
\hline 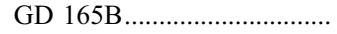 & DA4 & L4 & 3.7 & 120 & $\lesssim 1$ & $\lesssim 30$ & 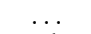 & $1.2-5.5$ & $3,4,5$ \\
\hline 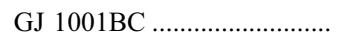 & M4 V & $\mathrm{L} 5+\mathrm{L} 5^{\mathrm{b}}$ & 18 & 170 & 0.09 & 0.8 & $\sim 1^{\mathrm{b}}$ & $1-10$ & 6,7 \\
\hline$\eta$ Tel B ${ }^{\mathrm{c}}$ & $\mathrm{A} 0 \mathrm{~V}$ & M7.5 V & 4.2 & 190 & $\lesssim 0.15$ & $\lesssim 7$ & $\ldots$ & $0.02-0.03$ & 8,9 \\
\hline GSC 08047-00232B …........... & K3 V & M6-9 V: & 3.2 & 200 & $\lesssim 0.1$ & $\lesssim 7$ & $\ldots$ & $0.01-0.04$ & $10,11,12$ \\
\hline GG Tau $\mathrm{Bb}^{\mathrm{d}} \ldots \ldots \ldots \ldots \ldots \ldots$ & M5 V & M7 V & 1.5 & 210 & $\lesssim 0.1$ & $\lesssim 14$ & ( & $0.01-0.02$ & 13,14 \\
\hline 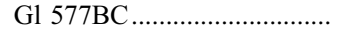 & G5 V & M5 V:+M5 V: & 5.4 & 240 & 0.08 & 3.6 & $\sim 1^{\mathrm{b}}$ & $0.3-0.5$ & $15,16,17,18$ \\
\hline GJ 1048B & $\mathrm{K} 2 \mathrm{~V}$ & L1 & 12 & 250 & $\lesssim 1$ & $\lesssim 20$ & $\ldots$ & $0.6-1.0$ & 19,20 \\
\hline G196-3B..... & $\mathrm{M} 2.5 \mathrm{~V}$ & L2 & 16 & 320 & $\lesssim 1$ & $\$ 20$ & $\ldots$ & $0.02-0.3$ & 21 \\
\hline GJ $4287 \mathrm{~B}^{\mathrm{f}} .$. & $\mathrm{M} 0 \mathrm{~V}+\mathrm{M} 0 \mathrm{~V}^{\mathrm{b}}$ & M9.5 V & 34 & 640 & $\lesssim 0.3$ & $\lesssim 6$ & $\ldots$ & $0.6-10$ & 22,23 \\
\hline LP $261-75 B^{g} \ldots \ldots \ldots \ldots \ldots \ldots \ldots \ldots \ldots \ldots \ldots \ldots$ & M: & L6 & 13 & 820 & $\lesssim 1$ & $\lesssim 60$ & $\ldots$ & $1-10$ & $24,25,26$ \\
\hline 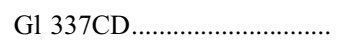 & $\mathrm{G} 8 \mathrm{~V}+\mathrm{K} 1 \mathrm{~V}$ & $\mathrm{~L} 8+\mathrm{L} 8 / \mathrm{T}:^{\mathrm{b}}$ & 43 & 880 & 0.53 & 10.9 & $\sim 1^{\mathrm{b}}$ & $0.6-3.4$ & 26,27 \\
\hline$\epsilon$ Ind BC & $\mathrm{K} 5 \mathrm{~V}$ & $\mathrm{~T} 1+\mathrm{T} 6$ & 402 & 1460 & 0.62 & 2.2 & $\sim 0.6$ & $0.8-2.0$ & 28,29 \\
\hline 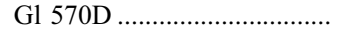 & $\mathrm{K} 4 \mathrm{~V}+\mathrm{M} 1.5 \mathrm{~V}+\mathrm{M} 3 \mathrm{~V}$ & T8 & 258 & 1530 & $\lesssim 0.1$ & $\lesssim 0.6$ & $\ldots$ & $2-5$ & 30,31 \\
\hline Gl 417BC & G0 V & L4.5+L6: ${ }^{\text {b }}$ & 90 & 2000 & 0.07 & 1.5 & $\sim 0.7^{\mathrm{b}}$ & $0.08-0.3$ & 32,33 \\
\hline 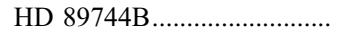 & F7 V/IV & L0 & 63 & 2460 & $\lesssim 1$ & $\lesssim 40$ & $\ldots$ & $1.5-3.0$ & 27,33 \\
\hline 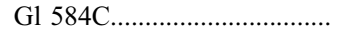 & $\mathrm{G} 1 \mathrm{~V}+\mathrm{G} 3 \mathrm{~V}$ & L8 & 194 & 3600 & $\lesssim 1$ & $\$ 20$ & $\ldots$ & $1.0-2.5$ & 32 \\
\hline
\end{tabular}

NoтE.-Widely separated brown dwarf companion systems are defined here as common proper or radial motion systems with star-brown dwarf separations greater than $100 \mathrm{AU}$.

${ }^{a}$ Upper limits on the apparent separations for equal-magnitude unresolved sources are taken from the literature or assumed to be 1 " in the case of no high-resolution imaging follow-up.

${ }_{\mathrm{b}}$ Estimated from differential photometry.

c Also known as HR 7329B (Lowrance et al. 2000).

d Also known as GG Tau/c, this pair is separated by $1400 \mathrm{AU}$ from the binary stellar pair GG Tau Aab. All four components share common radial motion and are believed to have formed from the same cloud core (White et al. 1999).

${ }^{\text {e }}$ Mugrauer et al. (2004) give a spectral type M4.5 for the combined system Gl 577BC, stating that it is a $0.16-0.20 M_{\odot}$ low-mass star. Lowrance et al. (2005) determine a spectral type of M5.5 and component masses of $0.08 M_{\odot}$. McCarthy et al. (2001) classify the source as M5 and at the stellar/substellar boundary. As such, we include this source as a possible double brown dwarf companion system.

${ }^{f}$ Also known as G216-7B (Kirkpatrick et al. 2001b).

${ }^{g}$ Also known as 2MASSW J0951054+355801 (Kirkpatrick et al. 2000), this object's proper motion as measured by Vrba et al. (2004), $\mu=00^{\prime \prime} 189 \pm 0.0^{\prime \prime} 011 \mathrm{yr}^{-1}$ at $\theta=211^{\circ} .8 \pm 1^{\circ} .6$, is consistent with that of LP $261-75$ from the revised New Luyten Two-Tenths catalog (Salim \& Gould 2003 ), $\mu=0.0^{\prime \prime} 203 \pm 0^{\prime \prime} 013 \mathrm{yr}^{-1}$ at $^{\circ}$ $\theta=209^{\circ} .9 \pm 1.5$. Given their close angular proximity (13."3) and common motion, these objects are likely to be gravitationally bound. The measured distance of LP 261-75B, $d=60 \pm 30 \mathrm{pc}$ (Vrba et al. 2004), places a poor constraint on the projected separation of this low-mass stellar-brown dwarf pair, but it is clearly a widely separated system.

References.-(1) Lowrance et al. 1999; (2) Neuhäuser et al. 2000; (3) Becklin \& Zuckerman 1988; (4) Kirkpatrick et al. 1993; (5) Kirkpatrick et al. 1999a; (6) Goldman et al. 1999; (7) Golimowski et al. 2004b; (8) Lowrance et al. 2000; (9) Guenther et al. 2001; (10) Chauvin et al. 2003; (11) Neuhäuser et al. 2003; (12) Neuhäuser \& Guenther 2004; (13) Leinert et al. 1991; (14) White et al. 1999; (15) Lowrance 2001; (16) McCarthy et al. 2001; (17) Mugrauer et al. 2004; (18) Lowrance et al. 2005; (19) Gizis et al. 2001; (20) Seifahrt et al. 2004; (21) Rebolo et al. 1998; (22) Kirkpatrick et al. 2001b; (23) A. J. Burgasser \& J. D. Kirkpatrick 2005, in preparation; (24) Kirkpatrick et al. 2000; (25) Vrba et al. 2004; (26) this paper; (27) Wilson et al. 2001; (28) Scholz et al. 2003; (29) McCaughrean et al. 2004; (30) Burgasser et al. 2000; (31) Burgasser et al. 2003; (32) Kirkpatrick et al. 2001a; (33) Bouy et al. 2003.

Burgasser et al. 2002b; Golimowski et al. 2004a). More surprisingly, early-type $\mathrm{T}$ dwarfs are generally brighter than latetype L dwarfs in the $1 \mu \mathrm{m}$ region (Dahn et al. 2002; Tinney et al. 2003; Vrba et al. 2004), indicating a significant redistribution of flux at near-constant luminosity. Cloud condensate models, which adequately reproduce the overall trends of the L-T transition (Ackerman \& Marley 2001; Marley et al. 2002; Tsuji 2002; Cooper et al. 2003), cannot reproduce these seemingly dramatic, apparently temperature-independent effects. More elaborate models, evoking cloud dissipation (Burgasser et al. 2002a) and variations in rainout efficiency (Knapp et al. 2004), have been proposed but remain controversial (Tsuji \& Nakajima 2003).

One complication in addressing this issue is the unknown diversity of physical properties (mass, age, metallicity) among the current population of field brown dwarfs. Differences among these parameters could potentially blur the fundamental physical process or processes governing the L-T transition. Late-type binaries such as Gl 337CD, whose presumably coeval components may straddle the L-T boundary, are crucial for studying this transition independent of differences in age or metallicity. Gl $337 \mathrm{CD}$ is a particularly vital case, as the physical parameters of the substellar components can be constrained by the properties of the stellar primaries. Resolved photometric and spectroscopic observations of this system may therefore help explain the changes that occur in brown dwarf atmospheres as they evolve from dusty $\mathrm{L}$ dwarfs to dust-free $\mathrm{T}$ dwarfs.

\section{SUMMARY}

We have resolved the companion L8 brown dwarf G1 337C using the Lick-LLNL AO system into a nearly equal magnitude, closely separated pair of cool brown dwarfs, hereafter named Gl $337 \mathrm{CD}$. The absence of a background source at the current position of Gl 337CD in either the original 2MASS or earlier POSS surveys implies common proper motion. The long (140$180 \mathrm{yr}$ ) orbit of this system is poorly suited for dynamical mass measurements, but its late spectral type makes it an important target for studying the L-T transition. G1 337CD joins a growing list of brown dwarf binaries that are widely separated but gravitationally bound to more massive stars, and we find evidence that the frequency of such binaries is higher than that seen among field brown dwarfs. Further study of these systems is warranted, as their orbital, physical, and atmospheric characteristics provide 
important clues to the formation and evolution of brown dwarfs and their cool atmospheres.

The authors would like to thank telescope operator $\mathrm{K}$. Chloros and AO operator E. Gates for their expert assistance during the observations and the University of California $\mathrm{Ob}$ servatories time allocation committee for its generosity. A. J. B. also thanks D. Koerner for help with the XPHOT program and acknowledges useful discussions with J. Gizis during the preparation of the manuscript. Support for this work was provided by NASA through the Spitzer Fellowship Program. This publication makes use of data from the Two Micron All Sky Survey, which is a joint project of the University of Massachusetts and the Infrared Processing and Analysis Center and funded by the National Aeronautics and Space Administration and the $\mathrm{Na}-$ tional Science Foundation. 2MASS data were obtained from the NASA/IPAC Infrared Science Archive, which is operated by the Jet Propulsion Laboratory, California Institute of Technology, under contract with the National Aeronautics and Space Administration. The authors thank the anonymous referee for her/his helpful critique of the original manuscript.
Ackerman, A. S., \& Marley, M. S. 2001, ApJ, 556, 872

Armitage, P. J., \& Bonnell, I. A. 2002, MNRAS, 330, L11

Barnaby, D., Spillar, E., Christou, J. C., \& Drummond, J. D. 2000, AJ, 119, 378

Bate, M. R., Bonnell, I. A., \& Bromm, V. 2002a, MNRAS, 332, L65

2002b, MNRAS, 336, 705

Bauman, B. J., et al. 1999, Proc. SPIE, 3762, 194

2002, Proc. SPIE, 4494, 19

Becklin, E. E., \& Zuckerman, B. 1988, Nature, 336, 656

Bouy, H., Brandner, W., Martín, E. L., Delfosse, X., Allard, F., \& Basri, G. 2003, AJ, 126, 1526

Burgasser, A. J., Kirkpatrick, J. D., Reid, I. N., Brown, M. E., Miskey, C. L., \& Gizis, J. E. 2003, ApJ, 586, 512

Burgasser, A. J., Marley, M. S., Ackerman, A. S., Saumon, D., Lodders, K., Dahn, C. C., Harris, H. C., \& Kirkpatrick, J. D. 2002a, ApJ, 571, L151

Burgasser, A. J., et al. 2000, ApJ, 531, L57

2002b, ApJ, 564, 421

Chauvin, G., et al. 2003, A\&A, 404, 157

Close, L. M., Siegler, N., Freed, M., \& Biller, B. 2003, ApJ, 587, 407

Cooper, C. S., Sudarsky, D., Milson, J. A., Lunine, J. I., \& Burrows, A. 2003, ApJ, 586, 1320

Cutri, R. M., et al. 2003, Explanatory Supplement to the 2MASS All Sky Data Release (Pasadena: Caltech), http://www.ipac.caltech.edu/2mass/releases/ allsky/doc/explsup.html

Dahn, C. C., et al. 2002, AJ, 124, 1170

Eggleton, P., \& Kiseleva, L. 1995, ApJ, 455, 640

Fischer, D. A., \& Marcy, G. W. 1992, ApJ, 396, 178

Forrest, W. J., Skrutskie, M. F., \& Shure, M. 1988, ApJ, 330, L119

Gavel, D. T., Olivier, S. S., Bauman, B. J., Max, C. E., \& Macintosh, B. A. 2000, Proc. SPIE, 4007, 63

Gizis, J. E., Kirkpatrick, J. D., \& Wilson, J. C. 2001, AJ, 121, 2185

Gizis, J. E., Monet, D. G., Reid, I. N., Kirkpatrick, J. D., Liebert, J., \& Williams, R. 2000, AJ, 120, 1085

Gizis, J. E., Reid, I. N., Knapp, G. R., Liebert, J., Kirkpatrick, J. D., Koerner, D. W., \& Burgasser, A. J. 2003, AJ, 125, 3302

Goldman, B., et al. 1999, A\&A, 351, L5

Golimowski, D. A., et al. 2004a, AJ, 127, 3516

2004b, AJ, 128, 1733

Goto, M., et al. 2002, ApJ, 567, L59

Guenther, E. W., Neuhäuser, R., Huélamo, N., Brandner, W., \& Alves, J. 2001, A\&A, 365, 514

Hayashi, C., \& Nakano, T. 1963, Prog. Theor. Phys., 30, 4

Hinz, J. L., McCarthy, D. W., Jr., Simons, D. A., Henry, T. J., Kirkpatrick, J. D., \& McGuire, P. C. 2002, AJ, 123, 2027

Kirkpatrick, J. D., Allard, F., Bida, T., Zuckerman, B., Becklin, E. E., Chabrier, G., \& Baraffe, I. 1999a, ApJ, 519, 834

Kirkpatrick, J. D., Dahn, C. C., Monet, D. G., Reid, I. N., Gizis, J. E., Liebert, J., \& Burgasser, A. J. 2001a, AJ, 121, 3235

Kirkpatrick, J. D., Kelly, D. M., Rieke, G. H., Liebert, J., Allard, F., \& Wehrse, R. 1993, ApJ, 402, 643

Kirkpatrick, J. D., Liebert, J., Cruz, K. L., Gizis, J. E., \& Reid, I. N. 2001b, PASP, 113, 814

Kirkpatrick, J. D., et al. 1999b, ApJ, 519, 802

. 2000, AJ, 120, 447

Knapp, G., et al. 2004, AJ, 127, 3553

Kumar, S. S. 1962, AJ, 67, 579

\section{REFERENCES}

Lane, B. F., Zapatero Osorio, M. R., Britton, M. C., Martín, E. L., \& Kulkarni, S. R. 2001, ApJ, 560, 390

Leinert, C., Haas, M., Mundt, R., Richichi, A., \& Zinnecker, H. 1991, A\&A, 250,407

Lloyd, J. P., Liu, M. C., Macintosh, B. A., Severson, S. A., Deich, W. T., \& Graham, J. R. 2000, Proc. SPIE, 4008, 814

Lowrance, P. J. 2001, Ph.D. thesis, Univ. California, Los Angeles

Lowrance, P. J., et al. 1999, ApJ, 512, L69

2000, ApJ, 541, 390

2005, ApJ, submitted

Luhman, K. L. 2004, ApJ, 614, 398

Marcy, G. W., \& Butler, R. P. 2000, PASP, 112, 137

Marley, M. S., Seager, S., Saumon, D., Lodders, K., Ackerman, A. S., Freedman, R., \& Fan, X. 2002, ApJ, 568, 335

Martín, E. L., Koresko, C. D., Kulkarni, S. R., Lane, B. F., \& Wizinowich, P. L. 2000, ApJ, 529, L37

Mason, B. D., McAllister, H. A., \& Hartkopf, W. I. 1996, AJ, 112, 276

McCarthy, C., Zuckerman, B., \& Becklin, E. E. 2001, AJ, 121, 3259

McCaughrean, M., Close, L. M., Scholz, R.-D., Lenzen, R., Biller, B., Brandner, W., Hartung, M., \& Lodieu, N. 2004, A\&A, 413, 1029

Monet, D. G., et al. 2003, AJ, 125, 984

Mugrauer, M., et al. 2004, A\&A, 417, 1031

Neuhäuser, R., \& Guenther, E. W. 2004, A\&A, 420, 647

Neuhäuser, R., Guenther, E. W., Alves, J., Huélamo, N., Ott, T., \& Eckart, A. 2003, Astron. Nachr., 324, 535

Neuhäuser, R., Guenther, E. W., Petr, M. G., Brandner, W., Huélamo, N., \& Alves, J. 2000, A\&A, 360, L39

Perryman, M. A. C., et al. 1997, The Hipparcos and Tycho Catalogues (ESA SP-1200; Noordwijk: ESA)

Potter, D., Martín, E. L., Cushing, M. C., Baudoz, P., Brandner, W., Guyon, O., \& Neuhäuser, R. 2002, ApJ, 567, L133

Pourbaix, D. 2000, A\&AS, 145, 215

Rebolo, R., Zapatero Osorio, M. R., Madruga, S., Béjar, V. J. S., Arribas, S., \& Licandro, J. 1998, Science, 282, 1309

Reid, I. N., Gizis, J. E., Kirkpatrick, J. D., \& Koerner, D. 2001, AJ, 121, 489

Reid, I. N., et al. 1991, PASP, 103, 661

Reipurth, B., \& Clarke, C. 2001, AJ, 122, 432

Richichi, A., Ragland, S., Calamai, G., Richter, S., \& Stecklum, B. 2000, A\&A, 361,594

Salim, S., \& Gould, A. 2003, ApJ, 582, 1011

Scholz, R.-D., McCaughrean, M. J., Lodieu, N., \& Kuhlbrodt, B. 2003, A\&A, 398, L29

Seifahrt, A., Neuhäuser, R., \& Mugrauer, M. 2004, A\&A, 421, 255

Shatsky, N., Sinachopoulos, D., Prado, P., \& van Dessel, E. 1999, A\&AS, 139, 69

Tinney, C. G., Burgasser, A. J., \& Kirkpatrick, J. D. 2003, AJ, 126, 975

Tokovinin, A. A. 1997, Astron. Lett., 23, 727

Tokovinin, A. A., \& Smekhov, M. G. 2002, A\&A, 382, 118

Tsuji, T. 2002, ApJ, 575, 264

Tsuji, T., \& Nakajima, T. 2003, ApJ, 585, L151

Vrba, F. J., et al. 2004, AJ, 127, 2948

White, R. D., Ghez, A. M., Reid, I. N., \& Schultz, G. 1999, ApJ, 520, 811

Wilson, A. G. 1952, Trans. IAU, 8, 335

Wilson, J. C., et al. 2001, AJ, 122, 1989

Zapatero Osorio, M. R., Lane, B. F., Pavlenko, Ya., Martín, E. L., Britton, M., \& Kulkarni, S. R. 2004, ApJ, 615, 958 